\title{
Nervous necrosis virus capsid protein exploits nucleolar phosphoprotein Nucleophosmin (B23) function for viral replication
}

\author{
Weijun Mai*; Fang Huang; Huiqing Chen; Yajing Zhou; Yan Chen \\ The Institute of Life Sciences, Jiangsu University, Zhenjiang, 212013, China \\ *Corresponding author. Tel: 86-0511-88791702; Fax: 86-0511-88791702; \\ *Corresponding author E-mail address: mwj9876@mail.ujs.edu.cn
}

Telephone number: 86-15262988928

\begin{abstract}
Nucleolar proteins facilitate the replication of certain human and animal viruses through interaction with viral proteins. In this study, an interaction between nervous necrosis virus capsid protein and nucleolar phosphoprotein B23 was identified using in vitro experimental approaches. The capsid protein binds to B23 early during the viral infection and accumulates in the nucleus, particularly in the nucleolus. However, over the course of the infection B23 is redistributed from the nucleoli to the nucleoplasm. siRNA-mediated knockdown of B23 reduced viral replication and cytopathic effect. Thus, B23 targets capsid protein to the nucleus and facilitates NNV replication. The results provide the first demonstration that nucleolar protein B23 has a direct role in the nodavirus replication process.
\end{abstract}

Keywords: Nervous necrosis virus; Capsid protein; Nucleolar protein B23; 
Interaction; Viral replication

\section{Introduction}

Viral nervous necrosis (VNN) disease, induced by the nervous necrosis virus (NNV), has caused mass mortality in cultured marine fish at the larval stage, resulting in significant economics losses (Munday et al., 2002; Chi et al., 2003). NNV targets the nervous system, and the characteristic pathological feature of VNN disease is vacuolation of the brain and retina (Munday et al., 1997). The NNV genome is composed of two positive single-stranded RNAs that lack poly-A tails. RNA1 encodes an RNA-dependent RNA polymerase (RdRp), and RNA2 encodes the capsid protein (Tan et al., 2001). A subgenome of RNA1, named RNA3, encodes the B1 and B2 proteins (Sommerset and Nerland, 2004). The B1 protein participates in anti-necrotic cell death by reducing the mitochondrial membrane potential (MMP) loss, and thus sustains cell viability (Chen et al., 2009). The B2 protein binds to newly synthesized viral double-stranded RNA, thereby preventing host RNA interference-mediated cleavage, and during the late stages of infection B2 can induce mitochondria-mediated cell death (Fenner et al., 2006; Su et al., 2014).

Nucleolar phosphoprotein Nucleophosmin (B23) is a ubiquitously expressed shuttling protein that participates in numerous cellular activities based on the phosphorylation, acetylation, ubiquitylation, and SUMOylation of its functional domains (Okuwaki, 2008). B23 has been demonstrated to bind both DNA and 
RNA, interact with several factors involved in the promotion of RNA processing, and prevent protein misfolding (Okuwaki et al., 2002; Lindström and Zhang, 2006). Recent studies have also highlighted that B23 is involved in additional nonclassical roles, including cell cycle regulation, cellular stress responses, and viral replication (Boisvert et al., 2007). Many viral proteins reportedly interact with B23, and are subsequently transported to the nucleus. Examples include the core protein of Hepatitis C virus (Mai et al., 2006), replication proteins of Adeno-associated virus (Bevington et al., 2007) and matrix protein of Newcastle disease virus (Duan et al., 2014). Furthermore, previous research has shown that various types of viruses can induce functional alterations in B23 that facilitate processes involved in viral infection, such as replication of viral DNA or RNA, viral assembly, and control of intracellular trafficking (Hiscox, 2002; 2007; Zakaryan and Stamminger, 2011).

Although B23 has an implicated role in mediating viral infection it is currently unknown if, and through which mechanism, nodavirus recruits B23. In order to investigate this we first performed a co-immunoprecipitation assay with extracts of NNV-infected cells to determine whether NNV capsid protein interacts with B23. Next, a glutathione-S-transferase (GST) pull-down assay was used to confirm a direct interaction. Additionally, we were able to demonstrate that NNV capsid protein accumulates in the nucleus by binding to B23 early in infection and that B23 is redistributed from the nucleolus to the nucleoplasm at a later point in the infection. Further characterization revealed that knockdown of B23 reduces the 
cytopathic effect (CPE) and replication efficiency of NVV. Therefore, the interaction of $\mathrm{B} 23$ with $\mathrm{NNV}$ capsid protein may represent an important mechanism in NNV replication.

\section{Materials and methods}

\subsection{Production of plasmids}

To generate his- and GST full-length NNV capsid proteins, PCR were performed to amplified ORF of NNV capsid gene from the full-length cDNA clone of NNV using specific primers (Table.1). The PCR product was then digested with restriction enzymes and inserted into pGEX6p-1 vector. The B23 open reading frame (ORF) was amplified by PCR using the cDNA of B23 of orange-spotted grouper Epinephelus coioides (Genbank accession number KU904400) as the template and then inserted into the pET28a and pGEX6p-1vectors using specific primers (Table 1).

\subsection{Cell lines and viruses}

The GS cells (Qin et al., 2006) were derived from spleen tissue of the grouper fish, and cultured at $28^{\circ} \mathrm{C}$ in Leibovitz's L15 medium supplemented with 5\% fetal bovine serum (FBS). RGNNV, a genotype of NNV, was selected for further study. The RGNNV-C strain, isolated from NNV-infected grouper (Epinephelus akaara), was used in this study (Liu et al., 2012). The virus was proliferated in GS cells with a multiplicity of infection (MOI) of 10, after which infected cells were incubated at $28^{\circ} \mathrm{C}$ in L-15 medium supplemented with 5\% FBS. Once a complete 
cytopathic effect (CPE) was observed the cells were centrifuged at 12,000 x $\mathrm{g}$ for 10 minutes, then the supernatant was collected for virus titration in the GS cells.

\subsection{Expression and purification of recombinant proteins}

The full-length recombinant His-B23 plasmid pET28a was expressed in E. coli BL21 and purified following Mai et al. (2014a). The full-length recombinant plasmids pGEX6p-1-capsid and pGEX6p-1-B23 were expressed in E. coli BL21 and purified using standard molecular procedures. All proteins used in the experiments were determined to have at least $95 \%$ purity by SDS-PAGE and Coomassie brilliant blue R-250 staining. Bovine serum albumin was used as the standard in the measurement of protein concentrations.

\subsection{Preparation of polyclonal antibody}

The antibody against B23 protein was prepared in-house for this study. Purified His-B23 protein was injected intradermally as an antigen to immunize a rabbit. The rabbit received injections once every 10 days over an eight-week period. For the first injection, $30 \mathrm{mg}$ of antigen was mixed with an equal volume of Freund's complete adjuvant (Sigma-Aldrich, St. Louis, MO). During the following three injections $30 \mathrm{mg}$ of antigen was mixed with an equal volume of Freund's incomplete adjuvant (Sigma-Aldrich). Four days after the final injection, the antiserum was collected from the rabbit through exsanguination. The titer count was determined to be 1:15,000 using an enzyme-linked immunosorbent assay (ELISA).

\subsection{Immunoprecipitation and immunoblotting}


The GS cells were infected with NNV (MOI of 10) for $24 \mathrm{~h}$, and total proteins were harvested using lysis buffer. Proteins extracted from noninfected GS cells and purified NNV were used as the controls. After centrifugation, the total proteins were incubated with anti-capsid (k233) or anti-B23 antibody for $2 \mathrm{~h}$. Immunoprecipitation (IP) and Western blotting (WB) assays were conducted using an ImmunoCruz IP/WB optima $F$ system (Santa Cruz) according to the manufacturer's instructions.

\subsection{GST pull-down assay}

GS cells were lysed in ice-cold lysis buffer (Takara, Shiga, Japan), after which 1 mg of cell extract was incubated with $10 \mu \mathrm{g}$ of GST-capsid, or GST together with glutathione-Sepharose, at $4^{\circ} \mathrm{C}$ for $3 \mathrm{~h}$. The beads were extensively washed in lysis buffer, size-fractionated by SDS-PAGE, and immunoblotted using anti-B23 antibody. Similarly, the GST pull-down assay using GST-B23 protein and exogenous his-capsid protein was performed as described above.

\subsection{Immunofluorescence imaging and analysis}

GS cells were infected with NNV at an MOI of 10 and prepared for Immunofluorescence analysis at $5 \mathrm{hpi}, 8 \mathrm{hpi}$ and $12 \mathrm{hpi}$, respectively. At the indicated times, cells were washed twice with PBS, fixed with $3.7 \%$ formaldehyde for $10 \mathrm{~min}$, and subjected to brief permeabilization with $0.5 \%$ Triton $\mathrm{X}-100$ in PBS. The cells were blocked for 30 min in PBS containing 10\% FCS and then incubated with anti-B23 and anti-capsid antibodies diluted in PBS containing 10\%

FCS for $1 \mathrm{~h}$. Next, the cover slips were washed three times with PBS and then 
incubated with either Alexa Fluor 488 goat anti-mouse immunoglobulin G or Alexa Fluor 594 goat anti-rabbit immunoglobulin G antibody (Invitrogen, Waltham, MA), as appropriate for the primary antibody used, for $1 \mathrm{~h}$. The cells were counterstained with DAPI to detect nuclei and imaged using a confocal laser scanning microscopy, TCS SP8 (Leica, Wetzlar, Germany).

\subsection{B23 RNA knockdown and virus infection}

B23 short-interfering RNAs (B23-siRNAs), negative siRNA control (Cat. no. 12935-400) and transfection reagents were purchased from Invitrogen. The siRNA sequences were listed in Table 1. To study the effect of B23 knockdown on the replication of NNV, GS cells were transfected with B23 siRNA for $24 \mathrm{~h}$ and then infected with $\mathrm{NNV}(\mathrm{MOI}=10)$. The cultured cells were collected at five time-points $(6,12,24,48,72 \mathrm{hpi})$, and virus titers were determined based on $50 \%$ tissue culture infective dose (TCID50) in GS cells. Total cellular RNA was extracted for Real-time reverse transcription (RT)-PCR analysis of the expression of NNV genes.

\subsection{Real-time quantitative PCR analysis}

To quantify B23 and NNV capsid protein expression levels, a SYBR green-based real-time PCR method (Takara) was used. GADPH mRNA was quantified to normalize total RNA concentrations in the samples. Table 1 lists the sequences of the primer sets used to amplify NNV target genes. RNA isolation and the real-time PCR operation were carried out according to Mai et al. (2014b). The standard curve method was used to determine fold-changes of capsid gene mRNA 
expression levels.

\subsection{Statistical analysis}

Statistical analyses were performed using PAWS Statistics 18 (SPSS Inc., Chicago, IL). The Independent-Samples T-test was used to determine significant differences between NNV-infected RNAi control cells and B23-RNAi cells. The same method was used to distinguish differences in NNV capsid mRNA expression levels and viral TCID50 values in cells with either knockdown or basal B23 levels. P-values $<0.01$ (marked by asterisks in the figures) were considered statistically significant.

\section{Results}

\subsection{NNV infection considerably affects B23 gene expression}

To analyze the effect of NNV infection on B23 gene expression, NNV-infected GS cells were collected at different time points, and the gene expression levels of both B23 and NNV capsid protein were detected through RT-PCR (Fig.1). Following NNV infection, B23 gene expression continuously increased from 0 to $12 \mathrm{hpi}$, with peak expression at $12 \mathrm{hpi}$. Expression began to decrease during 24-48 hpi. In contrast, expression of NNV capsid protein was first detected at 6 hpi, and increased from12-48 hpi. These results suggest that the increased capsid expression levels 6 hpi could be related to the increase in B23 expression up to 12 hpi.

\subsection{B23 and capsid protein interact in NNV-infected cells}


As B23 can shuttle between the cytoplasm and nucleus (Borer et al., 1989) and NNV capsid protein has also been found in both the cytoplasm and nucleus (Wu et al., 2010), we hypothesized that these two proteins may interact. To test whether B23 and capsid protein directly interact in vitro, GST pull-down assays were carried out using purified recombinant proteins (Fig. 2A). A protein binding assay of fusion GST-capsid protein to endogenous B23 protein showed that endogenous B23 was pulled-down by the GST-capsid protein but not by GST (Fig. 2A-a). Additionally, exogenous GST-B23 protein could bind to his-capsid protein, but not GST (Fig. 2A-b).Therefore, B23 and NNV capsid protein can directly interact in vitro.

To further confirm the interaction between capsid protein and B23 in GS cells, we performed Co- immunoprecipitation experiments. By western blot analysis, B23 and capsid proteins were both detected in the lysates of NNV-infected GS cells 24 hpi with anti- B23 and anti- capsid antibodies, respectively. (Fig. 2B, upper panel). As shown in the middle panel of Fig. 2B, Western blot analyses revealed that both $\mathrm{B} 23$ and NNV capsid protein coimmunoprecipitated with the anti-B23 antibody, confirming a physical interaction in virus-infected cells. The capsid protein-B23 interaction was further verified by the detection of capsid protein in a co-immunoprecipitation assay using anti-capsid antibody, as shown in the lower panel of Fig. 2B. These results demonstrate that NNV capsid protein physically interacts with B23 in vivo 
The interaction between B23 and NNV capsid protein indicated that the two proteins may share a similar intracellular localization. To test this possibility, we used confocal laser scanning microscopy to elucidate the two proteins' localization during the course of NNV infection. GS cells infected with NNV were fixed at different time points and the intracellular localization of capsid protein and B23 were examined by indirect immunofluorescence (Fig. 3). At 0 hpi, B23 protein was distributed in nucleus. At $5 \mathrm{hpi}$, the capsid protein was distributed in the whole cell, particularly in the nucleus, showing the same intracellular localization with B23 in the nucleus. At 8 hpi, the capsid protein was concentrated more in the nucleolus and B23 protein was redistributed in the nucleoplasm and the nucleus in addition to the nucleolus. At $12 \mathrm{hpi}$, the capsid protein was distributed in the whole cells and B23 protein was localized mainly in the nucleoplasm. All together, these results demonstrate that B23 co-localizes with capsid in the nucleus early in infection.

\subsection{B23 knokdown restricts capsid protein accumulation in the nucleus}

As B23 targets proteins to the nucleus, and NNV capsid protein interacts with B23, it seemed plausible that capsid protein accumulates in the nucleus by binding to B23. To test this hypothesis, the subcellular localization of capsid protein in B23-knokdown GS cells was investigated. Three pairs of synthesized B23 siRNAs were transfected into GS cells respectively, and one (B23 RNAi3) could effectively lower the expression level of B23 without causing discernable changes in cell morphology (Fig. 4A and B). In the present study, the capsid protein 
$\underline{\text { accumulation after B23 RNAi3 transfection was examined. As a result of B23 }}$ knokdown, capsid protein was localized mainly to the whole cell at $8 \mathrm{hpi}$, and nucleolar accumulation of capsid protein was markedly reduced compared to the RNAi control (Fig. 4C). These data confirm that capsid protein accumulates in the nucleus by binding to B23.

\subsection{Knockdown of B23 inhibits NNV replication in GS cells}

Since B23 plays an essential role in the nucleolar localization of capsid protein, this might be critical for the replication of NNV. To this end, NNV replication in GS cells with siRNA-mediated knockdown of B23 was investigated. As shown in Fig. 5A-C, knockdown of B23 markedly reduced NNV-induced CPE at 24 hpi, and was accompanied by a significant reduction of viral load in the cell culture, relative to RNAi controls ( $\mathrm{p}<0.01$ ) (Fig. 5D). To further determine effects of B23 knockdown on NNV replication, the mRNA expression levels of NNV capsid protein in NNV-infected B23-RNAi cells were examined. We found that the transcription level of NNV capsid protein was significantly lower in B23-RNAi cells was than in RNAi control, and normal cells ( $p<0.01)$ (Fig. 5E).This indicates that reduced levels of B23 slowed viral replication, suggesting an important role for B23 in NNV replication.

\section{Discussion}

The nucleolus is the most distinct structure in the cell nucleus, but its structure constantly changes due to changes in metabolic conditions. Some of the most challenging changes, structurally and functionally, are induced by viral infection 
(Hiscox, 2002; Greco, 2009; Harrison et al., 2010). In recent years, it has been shown that various types of viruses target nuclear, particularly nucleolar, proteins in both replication and pathogenic processes (Huang et al., 2001; Sirri et al., 2008; Harrison et al., 2010) Notably, the capsid protein of the nodavirus NNV reportedly interacts with grouper heat shock cognate protein 70 (GHSC70), which may act as a receptor mediating entry of NNV into cells (Chang and Chi, 2015). In contrast, the barramundi Mx protein (BMx) can inhibit viral RNA synthesis by mediating redistribution of viral $\mathrm{RdRp}$ to the perinuclear area for degradation (Wu et al., 2010). However, relatively little is known about the contribution of capsid-interacting nucleolar proteins to NNV replication.

In the present study, nucleolar protein B23 was identified as a NNV capsid-interacting factor. Confocal laser scanning microscopy illustrated that NNV capsid protein and B23 are both localized to the nucleus in addition to the nucleolus during early stages of viral infection. However, siRNA-mediated depletion of B23 resulted in accumulation of NNV capsid protein in the nucleus, demonstrating that NNV capsid may accumulate in the nucleus through a specific interaction with B23. B23 has been described as a hub protein, able to target both cellular and viral proteins to the nucleus (Li, 1997; Wang et al., 2011; Lindstrom, 2012; Du et al., 2014). It is a multifunctional protein that has nucleic acid-binding, ribonuclease and molecular chaperone activities (Huang et al., 2005).Several studies have demonstrated that the C-terminal nucleic acid-binding domain of B23 is involved in the recognition of pre-ribosomal particles through RNA binding, 
substrates for ribonuclease activity, and key proteins involved in the ribosome assembly process (Hingorani et al., 2000; Itahana et al., 2003; Huang et al., 2005). One previous study speculated that virus capsid protein localizes to the nucleus, specifically to the nucleolus, as part of mechanism that inhibits host ribosome assembly and protein synthesis (Aguilar et al., 2007). The interaction we found between NNV capsid protein and B23 could contribute to this inhibition, as the binding of capsid protein to B23 may disrupt the structure, and thus function of B23.

Several other studies have shown that the redistribution of B23 to other cellular locations during viral infection is important for the virus life cycle (Hiscox, 2002). For example, adeno-associated virus (AAV) capsid protein translocates B23 from nucleoli to the cytoplasm in AAV trafficking and transduction processes (Johnson and Samulski, 2009). Furthermore, Herpes simplex virus 1 (HSV-1) UL24 protein causes the dispersal of B23 to the cytoplasm, which promotes the nuclear egress of viral particles (Lymberopoulos et al., 2011). More recently, studies have demonstrated that adenoviral protein $\mathrm{V}$ promotes the $\mathrm{B} 23$ translocation from the nucleoli to the nucleoplasm, and this translocation is correlated with adenoviral replication (Ugai et al., 2012). Although the redistributions of B23 caused by different viruses have the difference, depletion of B23 reduces virus replication (Duan et al., 2014; Jeong et al., 2014). This redistribution was also noted in our experiments. During the early stages of infection B23 was localized to the nucleus, specifically to the nucleolus, but as the infection progressed B23 translocated to 
the nucleoplasm. In addition, the inhibition of B23 in the GS cells decreases extracellular NNV titers and the replication efficiency of NNV. These results suggest that inhibition of B23 might reduce intracellular capsid formation resulting in a decrease of NNV replication (Jeong et al., 2014).

To the best of our knowledge, this is the first report revealing that B23 is crucial in the early stage of NNV infection. We demonstrated that B23 facilitates NNV replication by transferring capsid protein to the nucleus via a direct capsid-B23 interaction. Although our studies demonstrated the importance of a capsid-B23 interaction for $\mathrm{NNV}$ replication, the precise role of $\mathrm{B} 23$ in $\mathrm{NNV}$ pathogenesis remains elusive. Further investigations focusing on proteomic changes and effects of modifying the NNV genome are required to elucidate the molecular basis of B23 involvement in NNV pathogenesis.

\section{Acknowledgements}

This research was supported by the National Science Foundation for Young Scientists of China (Grant Nos. 31001132 and 31370790), the Natural Science Foundation of the Higher Education Institutions of Jiangsu Province (Grant No. 09KJB180001) and the Startup Scientific Research Fund from Jiangsu University for Advanced Professionals (Grant No.10JDG075). We would like to thank the Director of the Guangdong Provincial Key Laboratory of Marine Biology Applications (LAMB) for providing the facilities to carry out this work and for valuable advice and suggestions during the experiments. 


\section{References}

Aguilar, P.V., Weaver, S.C., Basler, C.F., 2007. Capsid protein of eastern equine encephalitis virus inhibits host cell gene expression. J. Virol. 81, 3866-3876.

Bevington, J. M., Needham, P.G., Verrill, K.C., Collaco, R.F., Basrur, V., Trempe, J.P., 2007. Adeno-associated virus interactions with B23/Nucleophosmin: identification of sub-nucleolar virion regions. Virology 357, 102-113.

Boisvert, F.M., vanKoningsbruggen, S., Navascues, J., Lamond, A.I., 2007. The multifunctional nucleolus. Nat.Rev.Mol.Cell Biol. 8, 574-585.

Borer, R.A., Lehner, C.F., Eppenberger, H.M., Nigg, E.A., 1989. Major nucleolar proteins shuttle between nucleus and cytoplasm. Cell 56, 379-390.

Chang, J.S., Chi, S.C., 2015. GHSC70 is involved in the cellular entry of nervous necrosis virus. J. Virol. 89, 61-70.

Chen, L.J., Su, Y.C., Hong, J.R., 2009. Betanodavirus non-structural protein B1: A novel anti-necrotic death factor that modulates cell death in early replication cycle in fish cells. Virology 385, 444-454.

Chi, S.C., Shieh, J.R., Lin, S.J., 2003. Genetic and antigenic analysis of betanodaviruses isolated from aquatic organisms in Taiwan. Dis. Aquat. Organ. 55, 221-228.

Duan, Z., Chen, J., Xu, H., Zhu, J., Li, Q., He, L., Liu, H., Hu, S., Liu, X., 2014. The nucleolar phosphoprotein B23 targets Newcastle disease virus matrix protein to the nucleoli and facilitates viral replication. Virology. 452-453, $212-222$ 
Fenner, B.J., Thiagarajan, R., Chua, H.K., Kwang, J., 2006. Betanodavirus B2 is an RNA interference antagonist that facilitates intracellular viral RNA accumulation. J. Virol. 80, 85-94.

Greco, A., 2009. Involvement of the nucleolus in replication of human viruse. Rev. Med. Virol. 19, 201-214.

Harrison, M.S., Sakaguchi, T., Schmitt, A.P., 2010. Paramyxovirus assembly and budding: building particles that transmit infections. Int. J. Biochem. Cell Biol. $42,1416-1429$.

Hingorani, K., Szebeni, A., Olson, M.O., 2000. Mapping the functional domains of nucleolar protein B23. J. Biol. Chem. 275, 24451-24457.

Hiscox, J.A., 2002. The nucleolus - a gateway to viral infection? Arch. Virol. 147, 1077-1089.

Hiscox, J.A., 2007. RNA viruses: hijacking the dynamic nucleolus. Nat. Rev. Microbiol. 5, 119-127.

Huang, W.H., Yung, B.Y., Syu, W.J., Lee, Y.H., 2001. The nucleolar phosphoprotein B23 interacts with hepatitis delta antigens and modulates the hepatitis delta virus RNA replication. J. Biol. Chem. 276, 25166-25175.

Huang, N., Negi, S., Szebeni, A., Olson, M.O., 2005. Protein NPM3 interacts with the multifunctional nucleolar protein B23/nucleophosmin and inhibits ribosome biogenesis. J. Biol. Chem. 280, 5496-5502.

Itahana, K., Bhat, K.P., Jin, A., Itahana, Y., Hawke, D., Kobayashi, R., Zhang, Y., 2003. Tumor suppressor ARF degrades B23, a nucleolar protein involved in 
ribosome biogenesis and cell proliferation. Mol. Cell. 12, 1151-1164.

Jeong, H., Cho, M.H., Park, S.G., Jung, G., 2014. Interaction between nucleophosmin and HBV core protein increases HBV capsid assembly. FEBS Lett. 588, 851-858.

Johnson, J.S., Samulski, R.J., 2009. Enhancement of adeno-associated virus infection by mobilizing capsids into and out of the nucleolus. J. virol. 83, 2632-2644.

Li, Y.P., 1997. Protein B23 is an important human factor for the nucleolar localization of the human immunodeficiency virus protein. Tat. J. Virol. 71, 4098-4102.

Lindström, M.S., Zhang, Y., 2006. B23 and ARF: friends or foes? Cell Biochem. Biophys. 46, 79-90.

Lindstrom, M.S., 2012. Elucidation of motifs in ribosomal protein S9 that mediate its nucleolar localization and binding to NPM1/nucleophosmin. PLoS One 7, e52476.

Liu, H., Teng, Y., Zheng, X., Wu, Y., Xie, X., He, J., Ye, Y., Wu, Z., 2012. Complete sequence of a viral nervous necrosis virus (NNV) isolated from red-spotted grouper (Epinephelusakaara) in China. Arch. Virol. 157, 77-82.

Lymberopoulos, M.H., Bourget, A., Ben Abdeljelil, N., Pearson, A., 2011. Involvement of the UL24 protein in herpes simplex virus 1-induced dispersal of B23 and in nuclear egress. Virology 412, 341-348.

Mai, R.T., Yeh, T.S., Kao, C.F., Sun, S.K., Huang, H.H., Lee, Y.W., 2006. Hepatitis 
$\mathrm{C}$ virus core protein recruits nucleolar phosphoprotein B23 and coactivator p300 to relieve the repression effect of transcriptional factor YY1 on B23 gene expression. Oncogene, 25, 448-462.

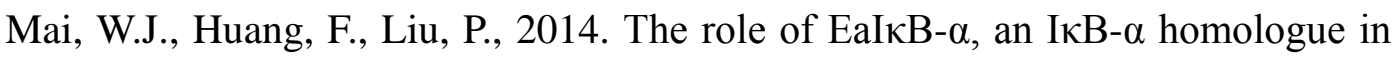
Epinephelusakaara, involved in innate immune response. Biotechnol. Lett. $36,1421-1429$.

Mai, W.J., Liu, P., Chen, H.Q., Zhou, Y.J., 2014. Cloning and immune characterization of the c-type lysozyme gene in red-spotted grouper, Epinephelus akaara. Fish Shellfish Immunol. 36, 305-314.

Munday, B.L., Kwang, J., Moody, N., 2002. Betanodavirus infection of teleost fish: a review. J. Fish. Dis. 25, 127-142.

Munday, B.L., Nakai, T., 1997. Nodaviruses as pathogens in larval and juvenile marine finfish. World J. Microb. Biot. 13, 375-381.

Okuwaki, M., Tsujimoto, M., Nagata, K., 2002. The RNA binding activity of a ribosome biogenesis factor, nucleophosmin/B23, is modulated by phosphorylation with a cell cycle-dependent kinase and by association with its subtype. Mol. Biol. Cell. 13, 2016-2030.

Okuwaki, M., 2008. The structure and functions of NPM1/nucleophsmin/B23, a multifunctional nucleolar acidic protein. J. Biochem. 143, 441-448.

Qin, Q.W., Wu, T.H., Jia, T.L., Hegde, A., Zhang, R.Q., 2006. Development and characterization of a new tropical marine fish cell line from grouper, Epinepheluscoioidessusceptible to iridovirus and nodavirus. J. Virol. Methods 
$131,58-64$.

Sirri, V., Urcuqui-Inchima, S., Roussel, P., Hernandez-Verdun, D., 2008. Nucleolus: the fascinating nuclear body. Histochem. Cell Biol. 129, 13-31.

Sommerset, I., Nerland, A.H., 2004. Complete sequence of RNA1 and subgenomic RNA3 of Atlantic halibut nodavirus (AHNV). Dis. Aquat. Org. 58, 117-125.

Su, Y.C., Chiu, H.W., Hung, J.C., Hong, J.R., 2014. Beta-nodavirus B2 protein induces hydrogen peroxide production, leading to Drp1-recruited mitochondrial fragmentation and cell death via mitochondrial targeting. Apoptosis 19, 1457-1470.

Tan, C., Huang, B., Chang, S.F., Ngoh, G.H., Munday, B., Chen, S.C., Kwang, J., 2001. Determination of the complete nucleotide sequences of RNA1 and RNA2 from greasy grouper (Epinephelustauvina) nervous necrosis virus, Singapore strain. J. Gen. Virol. 82, 647-653.

Ugai, H., Dobbins, G.C., Wang, M., Le, L.P., Matthews, D.A., Curiel, D.T., 2012. Adenoviral protein $\mathrm{V}$ promotes a process of viral assembly through nucleophosmin 1. Virology 432, 283-295.

Wang, Y., Chen, B., Li, Y., Zhou, D., Chen, S., 2011. PNRC accumulates in the nucleolus by interaction with B23/nucleophosmin via its nucleolar localization sequence. Biochim. Biophys. Acta 1813, 109-119.

Wu, Y.C., Lu, Y.F., Chi, S.C., 2010. Anti-viral mechanism of barramundi Mx against betanodavirus involves the inhibition of viral RNA synthesis through the interference of RdRp. Fish Shellfish Immunol. 28, 467-475. 
Zakaryan, H., Stamminger, T., 2011. Nuclear remodelling during viral infections. Cell Microbiol. 13, 806-813.

Fig.1. Real-time PCR analysis of gene expression levels B23 and NNV capsid during NNV infection. GS cells were infected with NNV (MOI=10), and subsequently collected at $6,12,24,36$ and 48 hours post infection (hpi). GADPH expression was used as the normalization standard. The Independent-Samples T-test was used to determine statistical significance $(*=p<0.01)$.

Fig.2. Interaction between NNV capsid protein and B23 in vitro and in vivo. (A) GS cell lysates were incubated with glutathione-sepharose beads bound to either GST (lane 3) or GST-capsid fusion protein (lane4). After washing, the bound proteins were separated by SDS-PAGE and transferred to a nitrocellulose membrane. (a) Endogenous B23 was detected by Western blotting using an anti-B23 polyclonal antibody. (b) His-tagged capsid protein was incubated with glutathione-sepharose beads bound to either GST (lane 3) or GST-B23 fusion protein (lane 4). After washing, the bound protein was detected using an anti-capsid antibody. (B) Results of co-immunoprecipitation assay testing interaction between his-B23 and capsid protein in GS cells. GS cells were infected with NNV (MOI=10). At 24 hpi cells were lysed, and a co-immunoprecipitation assay was performed using either anti-B23 (middle panel) or anti-capsid (lower panel) antibody. The input samples are shown in the upper panel. 
Fig.3. Intracellular distribution of B23 and NNV capsid protein. GS cells were infected with $\mathrm{NNV}(\mathrm{MOI}=10)$ and fixed at different time points $(5,8,12 \mathrm{hpi})$, then analyzed by immunofluorescence staining.

Fig.4. B23 knockdown restricts capsid protein accumulation in nuclei. (A) Effects of B23 RNAi on the expression of endogenous B23. GS cells were transfected with siRNA (1-3) or controls. After $48 \mathrm{~h}$ transfection, cell lysates were prepared and examined by Western blot with anti-B23 antibody. Endogenous_GADPH expressions were used as internal controls. (B) The relative levels of B23 in B23 siRNA-treated cells. The density of bands in (A) was quantitated by densitometry. The relative levels of B23 were calculated from three independent experiments performed as follows: the band density of B23/the band density of GADPH, with standard deviations indicated by error bars. (C) GS cells were transfected with control siRNA or B23 siRNA. 48 hours after transfection, cells were infected with NNV (MOI=10). At 8 hpi, the infected cells were stained by incubation with either anti-capsid protein or anti-B23 antibody, followed by a secondary antibody with a conjugated fluorescent probe. Nuclei were stained with DAPI. The subcellular localization of capsid protein and B23 was visualized at a magnification of 200x

Fig.5. Knockdown of B23 inhibits NNV replication in GS cells. Morphological changes in normal (A), RNAi control (B), and B23-RNAi (C) GS cells following 
NNV infection (MOI=10). (D) Knockdown of B23 reduced NNV growth. Normal, RNAi control, and B23-RNAi cells were infected with NNV (MOI=10). At five time points $(6,12,24,48$ and 72 hpi), the viral load in GS cell culture was determined in terms of TCID50, shown as average viral titers from three independent experiments.(E) Knockdown of B23 reduced expression of NNV capsid protein. Normal, RNAi control, and B23-RNAi cells were infected with NNV (MOI=10). 24 hours after NNV infection, the cells were examined by real-time RT-PCR to determine the expression level of NNV capsid protein. The graph shows average mRNA levels of NNV capsid protein over three independent experiments. An Independent-Samples T-Test was used to determine the significance $(*=\mathrm{p}<0.01)$ of differences between $\mathrm{B} 23-\mathrm{RNAi}$ and control RNAi cells. 


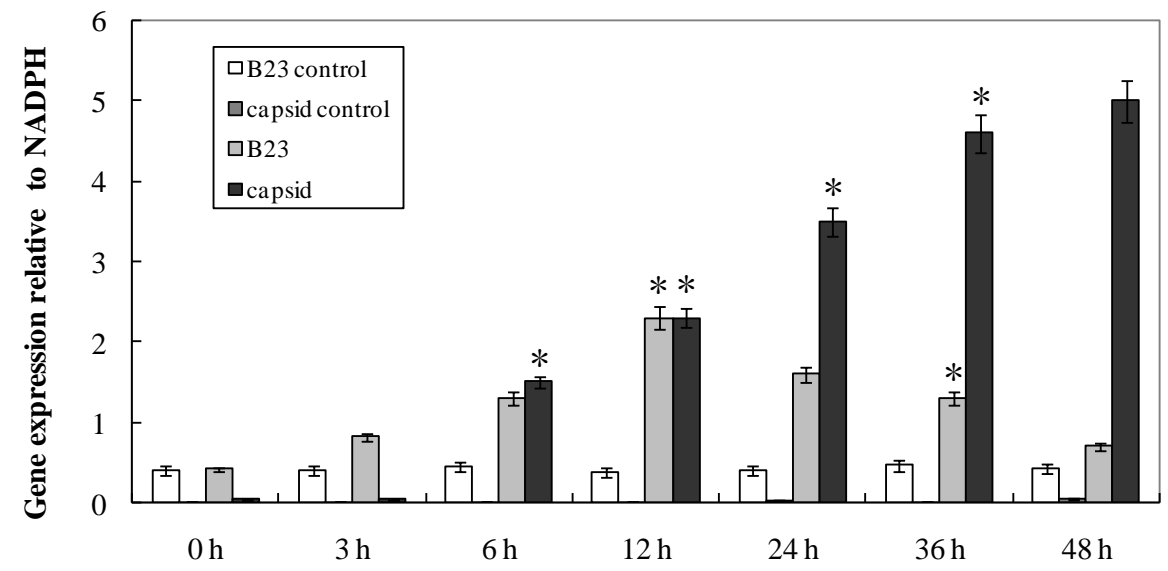

Fig.1

(A)

(a)

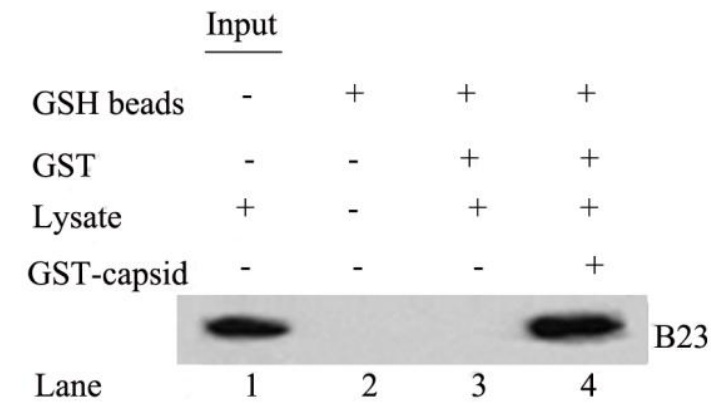

(b)

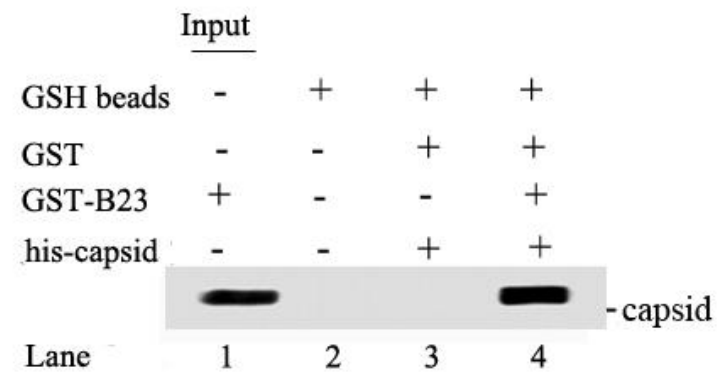

(B) 


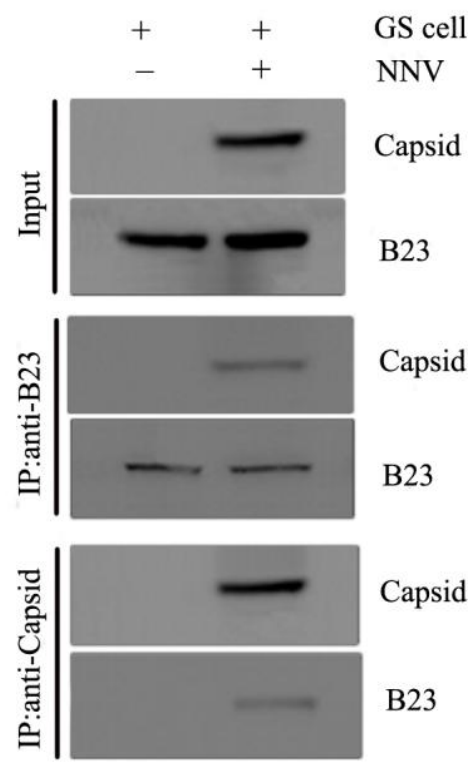

Fig.2

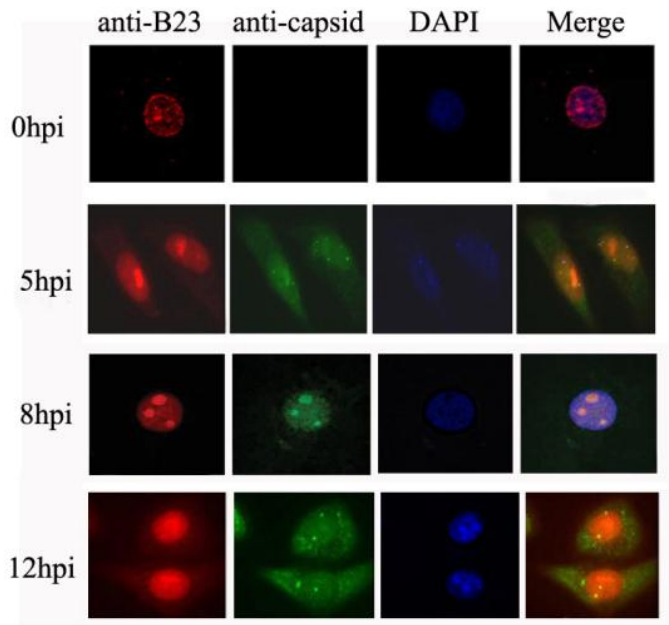

Fig.3

(A)

Control RNAi 1 RNAi 2 RNAi 3

WB: anti- B23

WB: anti-GADPH

(B) 


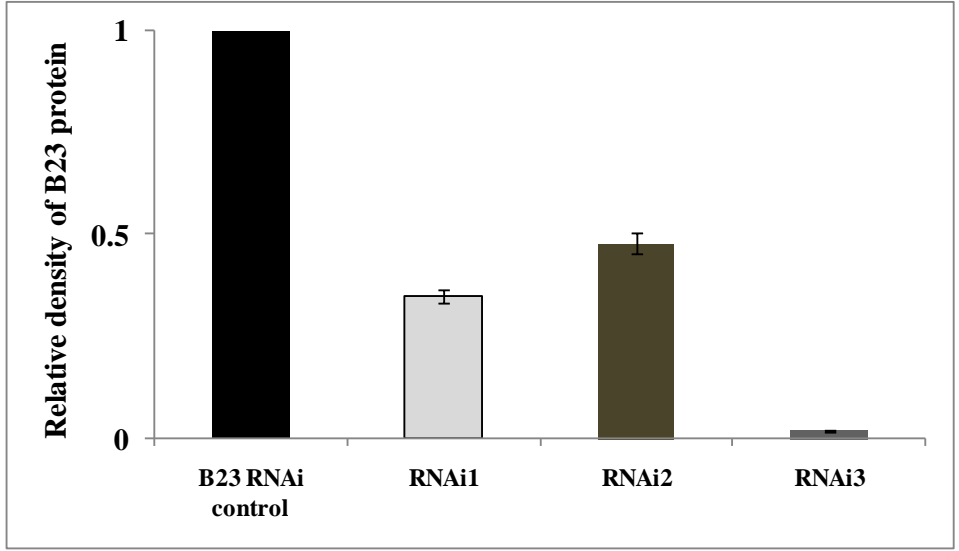

(C)

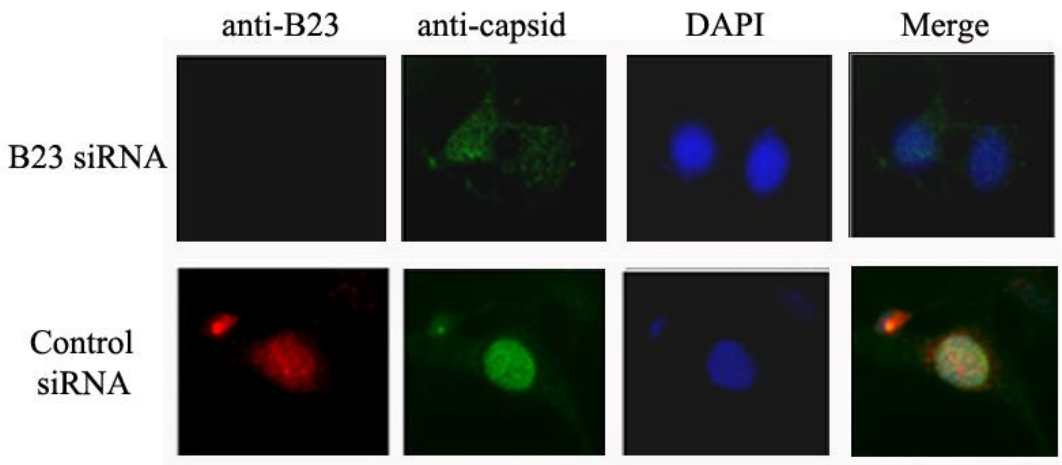

Fig. 4

Nomal cell

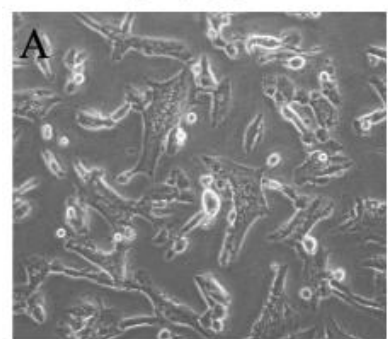

(D)

RNAi Control

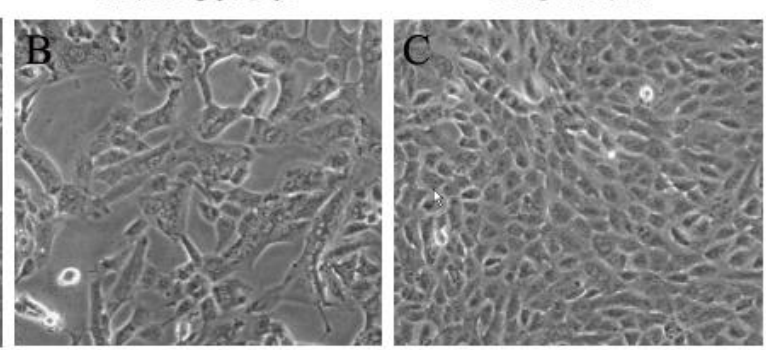




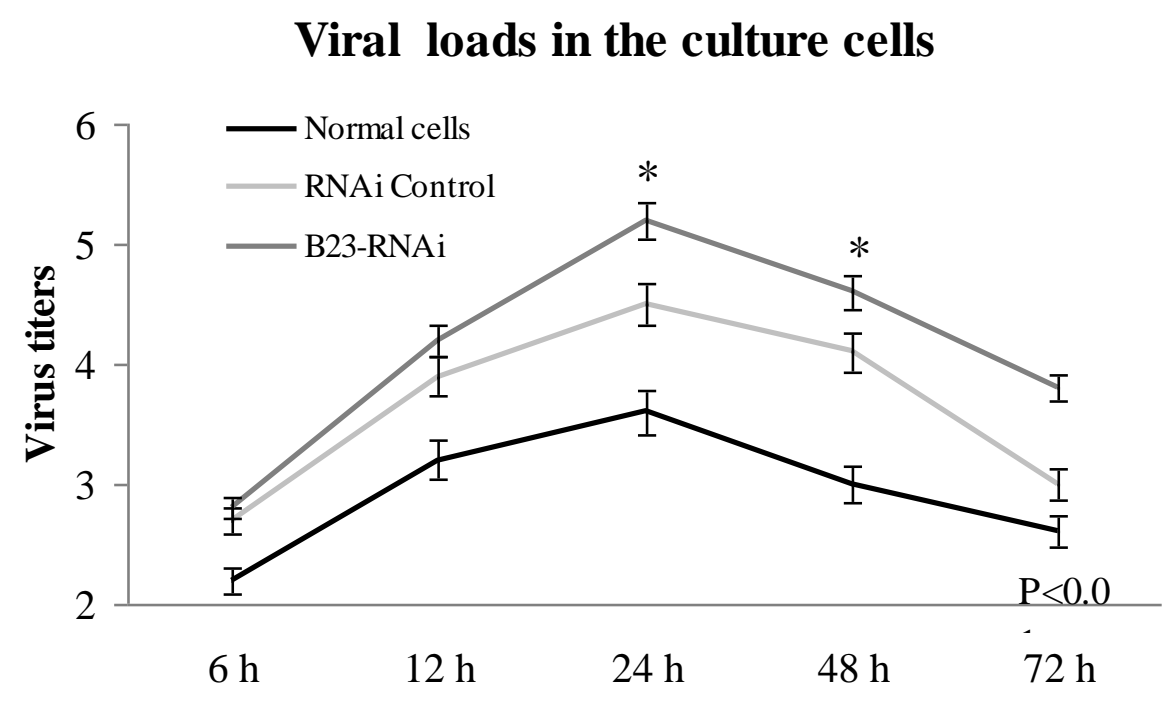

Hours post infection

(E)

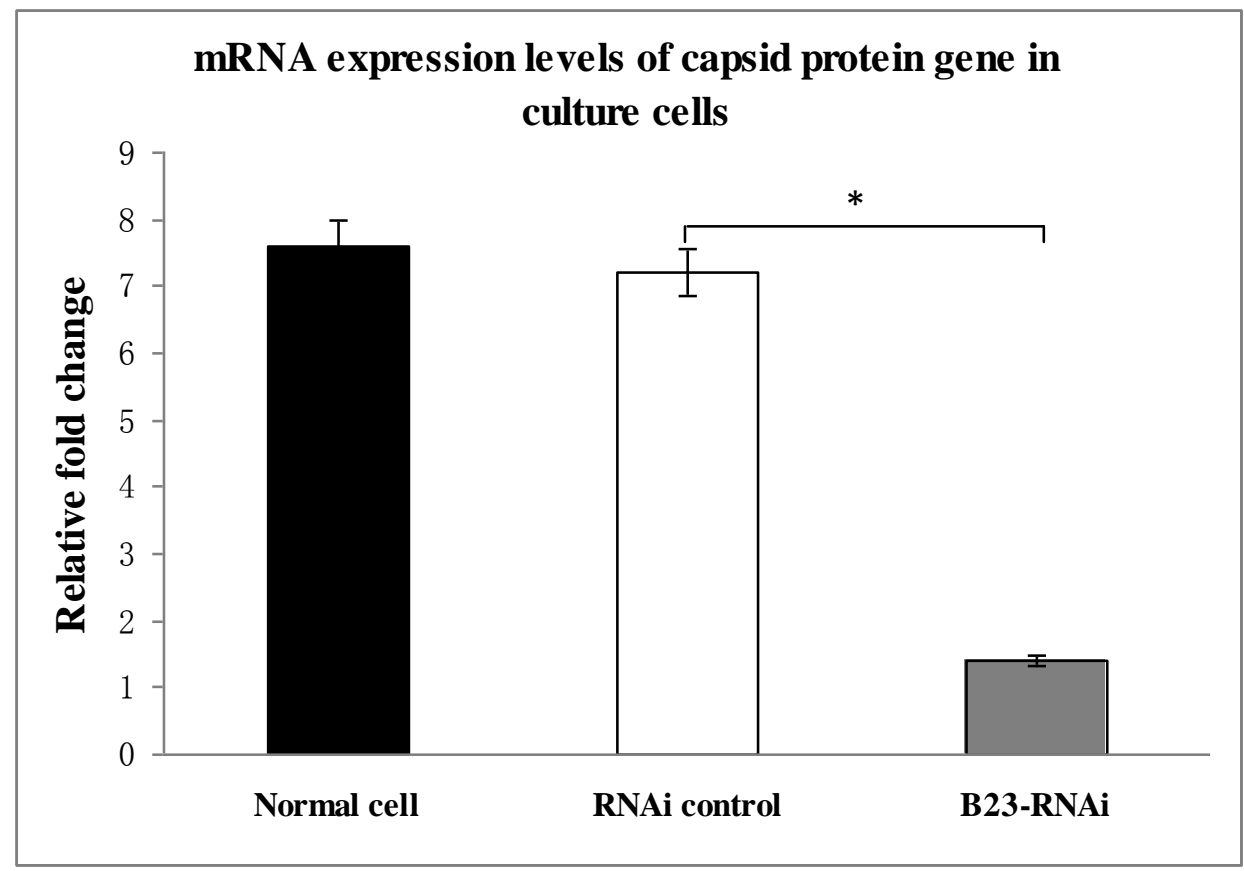

Fig.5 
Table.1. Primers and siRNA sequences used in this study

\begin{tabular}{ll}
\hline Primer name & Sequences (5'-3') \\
\hline Capsid-HisF & CGGGGATCCGACGATAGTCATGCCCCGCG \\
\hline Capsid-HisR & CGAGCGGCCGCAAGCTTCCATGGTACGCAAAG \\
\hline Capsid-GSTF & CGGGGATCCACCATG GCTAGA GGTAAACAAAAT \\
Capsid-GSTR & CGAGCGGCCGCATTATTGCCGACGATAGCTCT \\
B23-HisF & GACGACAAG GGATCCAGAAGGTGCGTCCCTGCAT \\
B23-HisR & GCTC GCGGCCGC-CTGACAGCGCCTCCAACAC \\
B23-GSTF & GACGACAAG GGATCCGAAGATTCGGATGGACA \\
B23-GSTR & GCTC GCGGCCGCTTAAAGAGACTTCCTCCACTGC \\
Capsid-RT-F & GCGCGTCGACATGGTACGCAAAGGTGA \\
Capsid-RT-R & GCGCGCAAGCTTTTAGTTTCCGAGTC \\
B23-RT-F & TAAGGATCCTTAACCACCTTTTTCTATAC \\
B23-RT-R & GCCTAAGGATCCTTAGCCGGCAGCCGA \\
GADPH-RT-F & ATCACAGCCACACAGAAGACGG \\
GADPH-RT-R & CTTTCCCCACAGCCTTAGCAGC \\
B23-RNAi1 & CAGUUUCACUAGGUGGAUUUGAGAU \\
B23-RNAi2 & GAGCCAAAGACGAAUUACAUGUUGU \\
B23-RNAi3 & CACCACCAUUUGUCUUGAGGUUAAA \\
Control siRNA & AUCUCAAAUCCACCUAGUGAAACUG \\
\hline
\end{tabular}

